\title{
Hypersurfaces of a Sasakian manifold - revisited
}

\author{
Sharief Deshmukh¹, Olga Belova², Nasser Bin Turki ${ }^{1}$ and Gabriel-Eduard Vîlcu ${ }^{3 *}$ (D)
}

\section{"Correspondence:}

gvilcu@upg-ploiesti.ro

${ }^{3}$ Department of Cybernetics, Economic Informatics, Finance and

Accountancy, Petroleum-Gas University of Ploieşti, Bd. Bucureşti

39, 100680 Ploieşti, Romania

Full list of author information is

available at the end of the article

\begin{abstract}
We study orientable hypersurfaces in a Sasakian manifold. The structure vector field $\xi$ of a Sasakian manifold determines a vector field $\mathbf{v}$ on a hypersurface that is the component of the Reeb vector field $\xi$ tangential to the hypersurface, and it also gives rise to a smooth function $\sigma$ on the hypersurface, namely the projection of $\xi$ on the unit normal vector field $\mathrm{N}$. Moreover, we have a second vector field tangent to the hypersurface, given by $\mathbf{u}=-\varphi(N)$. In this paper, we first find a necessary and sufficient condition for a compact orientable hypersurface to be totally umbilical. Then, with the assumption that the vector field $\mathbf{u}$ is an eigenvector of the Laplace operator, we find a necessary condition for a compact orientable hypersurface to be isometric to a sphere. It is shown that the converse of this result holds, provided that the Sasakian manifold is the odd dimensional sphere $\mathbf{S}^{2 n+1}$. Similar results are obtained for the vector field $\mathbf{v}$ under the hypothesis that this is an eigenvector of the Laplace operator. Also, we use a bound on the integral of the Ricci curvature $\operatorname{Ric}(\mathbf{u}, \mathbf{u})$ of the compact hypersurface to find a necessary condition for the hypersurface to be isometric to a sphere. We show that this condition is also sufficient if the Sasakian manifold is $\mathbf{S}^{2 n+1}$.
\end{abstract}

MSC: 53C42; 53C25; 53B25

Keywords: Hypersurface; Sasakian manifold; Laplace operator; Ricci curvature

\section{Introduction}

Sasakian manifolds are the odd dimensional counterpart to Kähler manifolds and, as pointed out recently in [24], many famous results from Kähler geometry were extended afterwards to Sasakian geometry (see, e.g., [12, 14, 19, 23, 26, 30, 41, 43]). Moreover, it is well known that Sasakian manifolds exist in abundance; for example the unit sphere $\mathbf{S}^{2 n+1}$, the Euclidean space $\mathbf{R}^{2 n+1}$, the unit tangent bundle $T_{1} \mathbf{S}^{2 n}$ of the unit sphere $\mathbf{S}^{2 n}$, the special unitary group $\mathbf{S U}(\mathbf{2})$, the Heisenberg group $\mathfrak{H}^{2 n+1}$ and the special linear group $\mathbf{S L}(\mathbf{2}, \mathbf{R})$ are Sasakian manifolds (cf. [15]). Recently, Boyer and Tonnesen-Friedman [16] constructed new explicit Sasakian metrics of constant scalar curvature on compact Sasakian manifolds of dimension $(2 n+3)$ which can be realized as certain 3-dimensional Lens space bundles over compact constant scalar curvature Kähler manifolds of real dimension $2 n$. Moreover, given a $(2 n+1)$-dimensional Riemannian manifold $(M, g)$, there is an associated Riemannian cone $M \times \mathbf{R}^{+}$with metric $\bar{g}=d t^{2}+t^{2} g$ and a smooth 1-form $\theta$ on $M$ is a contact form if and only if the 2-form $t^{2} d \theta+d t \wedge \theta$ on the associated cone is symplectic. Also, the

(c) The Author(s) 2021. This article is licensed under a Creative Commons Attribution 4.0 International License, which permits use, sharing, adaptation, distribution and reproduction in any medium or format, as long as you give appropriate credit to the original author(s) and the source, provide a link to the Creative Commons licence, and indicate if changes were made. The images or other third party material in this article are included in the article's Creative Commons licence, unless indicated otherwise in a credit line to the material. If material is not included in the article's Creative Commons licence and your intended use is not permitted by statutory regulation or exceeds the permitted use, you will need to obtain permission directly from the copyright holder. To view a copy of this licence, visit http://creativecommons.org/licenses/by/4.0/. 
associated cone is a Kähler manifold with Kähler form $t^{2} d \theta+d t \wedge \theta$ if and only if $(M, g)$ is a Sasakian manifold. This relationship between Sasakian manifolds and Kähler manifolds makes the geometry of Sasakian manifolds very interesting. In addition, it is well known that Sasakian manifolds play an important role in theoretical physics [15, 29, 40].

One of the most important branches of differential geometry is the geometry of submanifolds. In submanifold theory, it is more convenient to study submanifolds embedded in space forms (such as real, complex, quaternionic or Sasakian space forms), due to the fact that in all these ambient spaces, the fundamental equations take a simpler form (see, e.g., the monographs $[17,18]$ and $[2-5,39,42])$.

However, unlike the case of hypersurfaces in complex space forms which have been intensively studied (see, e.g., $[1,8,21,22,31,33,34,38,44])$ and despite the fact that Sasakian spaces are linked in a natural way to Kähler spaces, the geometry of hypersurfaces in Sasakian manifolds was not investigated with the same fervency, except some studies on hypersurfaces of Sasakian space forms (see [25]). In this setting, a remarkable result was obtained by Watanabe (cf. [45]), who used the famous Obata equation [35, 36] in proving that any complete and connected totally umbilical hypersurface of a $(2 n+1)$-dimensional Sasakian manifold of constant mean curvature $H$ is isometric to the sphere $\mathbf{S}^{2 n}\left(1+H^{2}\right)$. Note that Watanabe's study was further generalized in $[44,46]$.

If $M$ is an orientable hypersurface of a Sasakian manifold $\bar{M}(\varphi, \xi, \eta, g)$ of dimension $(2 n+1)$, with unit normal vector field $N$ and shape operator $A$, then one can consider two key vector fields on $M$, denoted by $\mathbf{v}$ and $\mathbf{u}$, where $\mathbf{v}$ is the tangential component of the Reeb vector field $\xi$ to $M$ and $\varphi(N)=-\mathbf{u}$. Recall now that the classical investigations of real hypersurfaces in complex space forms, as well as of hypersurfaces of Sasakian space forms were convenient due to a handy form of Gauss fundamental equation for the curvature tensor field and also due to a manageable form of Codazzi fundamental equation, but this tool is missing in the setting of hypersurfaces in general Sasakian manifolds. However, as proved in [6], this deficiency is compensated with the help of Reeb vector field $\xi$ of the Sasakian manifold and by the volume of the hypersurface. In Sect. 2, we state some basic formulae for later use, concerning orientable hypersurfaces $M$ of Sasakian manifolds $\bar{M}(\varphi, \xi, \eta, g)$ of dimension $(2 n+1)$. As totally umbilical hypersurfaces are important ones among hypersurfaces of Sasakian manifolds, in Sect. 3 we find the necessary and sufficient conditions for a compact orientable hypersurface to be a totally umbilical hypersurface (cf. Theorem 3.1). It is well known that the class of constant mean curvature hypersurfaces is a subject worthy of investigation in submanifold geometry (see, e.g., the recent articles $[7,9,11,20,27,28,37])$. These hypersurfaces, mostly known as CMC-hypersurfaces, have been investigated in various ambient spaces, including the unit sphere. As the odd dimensional sphere $\mathbf{S}^{2 n+1}$ is a remarkable example of Sasakian manifold, we use the tools developed in this paper to find necessary and sufficient conditions for a compact hypersurface of $\mathbf{S}^{2 n+1}$ to be a CMC-hypersurface (cf. Theorem 3.2). Moreover, we also find a necessary and sufficient condition for a compact hypersurface of $\mathbf{S}^{2 n+1}$ to be a small sphere (cf. Theorem 3.3).

In Sect. 4, we observe that, for a totally geodesic hypersurface $M$ of a $(2 n+1)$ dimensional Sasakian manifold $\bar{M}(\varphi, \xi, \eta, g)$, vector fields $\mathbf{u}, \mathbf{v}$ are eigenvectors of Laplace operator acting on smooth vector fields with eigenvalues 1 and $2 n-1$, respectively. This raises a natural question as to whether compact hypersurfaces for which these vector fields 
are eigenvectors with positive eigenvalues necessarily imply that hypersurfaces are totally geodesic? We answer this question in this section (cf. Theorem 4.1 and Theorem 4.3).

Finally, in Sect. 5, we use a lower bound on the integral of Ricci curvature $\operatorname{Ric}(\mathbf{u}, \mathbf{u})$ to find a condition under which a compact hypersurface is isometric to a sphere (cf. Theorem 5.2) and prove that this condition is also sufficient for hypersurfaces of the sphere $\mathbf{S}^{2 n+1}$ (cf. Theorem 5.3). We also use the restriction of the Ricci curvature $\overline{\operatorname{Ric}}(N, N)$ of the ambient Sasakian manifold to the compact orientable hypersurface $M$, which has an infimum $c$ (owing to compactness of $M$ ) and prove that, with suitable restrictions on $c$ and the sectional curvature of plane section spanned by $\mathbf{u}, \mathbf{v}$, there can be given a necessary condition for the hypersurface to be isometric to a sphere (cf. Theorem 5.4). We use this result to find the necessary and sufficient conditions for a compact hypersurface $M$ of the sphere $\mathbf{S}^{2 n+1}$ to be isometric to a small sphere (cf. Corollary 5.5).

\section{Preliminaries}

If $\bar{M}(\varphi, \xi, \eta, g)$ is a Sasakian manifold of dimension $(2 n+1)$ and $\bar{\nabla}$ denotes the Levi-Civita connection of the metric $g$, then it is well known that $\varphi$ is a tensor of type $(1,1)$ on $\bar{M}$, $\xi$ is a vector field on $\bar{M}$, called the Reeb vector field, the structure vector field or the characteristic vector field, $\eta$ is a 1-form on $\bar{M}, g$ is a Riemannian metric on $\bar{M}$, and the following relations are satisfied for all $X, Y \in \mathfrak{X}(\bar{M})[13,15]$ :

$$
\begin{aligned}
& \varphi^{2}(X)=-X+\eta(X) \xi, \quad \varphi(\xi)=0, \quad \eta \circ \varphi=0, \quad g(X, \xi)=\eta(X), \\
& g(\varphi(X), \varphi(Y))=g(X, Y)-\eta(X) \eta(Y), \\
& (\bar{\nabla} \varphi)(X, Y)=g(X, Y) \xi-\eta(Y) X, \quad \bar{\nabla}_{X} \xi=-\varphi X,
\end{aligned}
$$

where $\mathfrak{X}(\bar{M})$ denotes the Lie algebra of smooth vector fields on $\bar{M}$ and the covariant derivative $\bar{\nabla} \varphi$ of $\varphi$ is given by

$$
(\bar{\nabla} \varphi)(X, Y)=\bar{\nabla}_{X} \varphi(Y)-\varphi\left(\bar{\nabla}_{X} Y\right)
$$

As per usual, we denote in the following by $\bar{R}, \overline{\text { Ric }}$ and $\bar{Q}$, the curvature tensor, the Ricci tensor and the Ricci operator of $\bar{M}(\varphi, \xi, \eta, g)$. Then it is well known that [13]

$$
\bar{R}(X, Y) \xi=\eta(Y) X-\eta(X) Y, \quad \bar{Q}(\xi)=2 n \xi, \quad X, Y \in \mathfrak{X}(\bar{M}),
$$

where the Ricci operator $\bar{Q}$ is a symmetric operator defined with the help of the Ricci tensor $\overline{\text { Ric }}$ by

$$
\overline{\operatorname{Ric}}(X, Y)=g(\bar{Q}(X), Y) \text {. }
$$

Moreover, it is well known that, for all $X, Y$ orthogonal to $\xi$, we have

$$
\overline{\operatorname{Ric}}(\varphi(X), \varphi(Y))=\overline{\operatorname{Ric}}(X, Y), \quad X, Y \in \mathfrak{X}(\bar{M}) .
$$

If $M$ is an orientable hypersurface of the Sasakian manifold $\bar{M}(\varphi, \xi, \eta, g)$, let us denote by $N$ the unit normal vector field, and by $A$ the shape operator. Then the Gauss and Wein- 
garten basic formulae are written as (cf. [10, 17])

$$
\bar{\nabla}_{U} V=\nabla_{U} V+g(A U, V) N, \quad \bar{\nabla}_{U} N=-A U, \quad U, V \in \mathfrak{X}(M),
$$

where $g$ denotes the induced metric on $M$, as well as the metric on $\bar{M}, \nabla$ is the Levi-Civita connection of $M$ and $\mathfrak{X}(M)$ is the Lie algebra of the smooth vector fields on hypersurface $M$.

Now, due to the skew-symmetry of $\varphi$, we find that $\varphi(N)$ is orthogonal to $N$ and we obtain a smooth vector field $\mathbf{u} \in \mathfrak{X}(M)$ given by $\varphi(N)=-\mathbf{u}$. Let $\alpha$ be the smooth 1 -form on hypersurface $M$ dual to the vector field $\mathbf{u}$. Hence $\alpha(U)=g(\mathbf{u}, U), U \in \mathfrak{X}(M)$. Also, we consider an operator $F: \mathfrak{X}(M) \rightarrow \mathfrak{X}(M)$ defined by $F(U)=[\varphi(U)]^{T}$ the tangential component of $\varphi(U)$ to the hypersurface $M$. Therefore, we have

$$
\varphi(U)=F(U)+\alpha(U) N, \quad U \in \mathfrak{X}(M)
$$

and it follows immediately that $F$ is also a skew-symmetric operator.

Next, we define a smooth function $\sigma$ on $M$ by $\sigma=g(\xi, N)$. Then, on the hypersurface, we have

$$
\xi=\mathbf{v}+\sigma N
$$

where $\mathbf{v} \in \mathfrak{X}(M)$ is the tangential component of the Reeb vector field $\xi$. Let us denote by $\beta$ the smooth 1-form on $M$ dual to the vector field $\mathbf{v}$, that is, $\beta(U)=g(\mathbf{v}, U)$. Then, making use of $\varphi(N)=-\mathbf{u}$ and Eqs. (1), (2), (6), and (7), it follows that

$$
\begin{aligned}
& \|\mathbf{u}\|^{2}=\|\mathbf{v}\|^{2}=1-\sigma^{2}, \\
& F(\mathbf{u})=-\sigma \mathbf{v}, \quad F(\mathbf{v})=\sigma \mathbf{u}, \\
& F^{2}(U)=-U+\alpha(U) \mathbf{u}+\beta(U) \mathbf{v},
\end{aligned}
$$

and

$$
g(F(U), F(V))=g(U, V)-\alpha(U) \alpha(V)-\beta(U) \beta(V), \quad U, V \in \mathfrak{X}(M) .
$$

Also, making use of Eqs. (3), (4), (7), and $\varphi(N)=-\mathbf{u}$, we derive

$$
\begin{aligned}
& \nabla_{U} \mathbf{u}=\sigma U+F(A U), \quad \nabla_{U} \mathbf{v}=-F(U)+\sigma A U, \quad \nabla \sigma=-A \mathbf{v}-\mathbf{u}, \\
& (\nabla F)(U, V)=g(U, V) \mathbf{v}-\beta(V) U+\alpha(V) A U-g(A U, V) \mathbf{u},
\end{aligned}
$$

where $\nabla \sigma$ stands for the gradient of $\sigma$ and

$$
(\nabla F)(U, V)=\nabla_{U} F(V)-F\left(\nabla_{U} V\right)
$$

for $U, V \in \mathfrak{X}(M)$.

As $F$ is skew-symmetric and $A$ is symmetric, we deduce easily that

$$
\operatorname{tr}(F \circ A)=0,
$$


and using first two equations in (12), we derive

$$
\operatorname{div} \mathbf{u}=2 n \sigma, \quad \operatorname{div} \mathbf{v}=2 n H \sigma,
$$

where $H$ denotes the mean curvature of $M$ defined by $2 n H=t r A$. Thus, in the case that $M$ is compact, Eq. (14) implies

$$
\int_{M} \sigma=0, \quad \int_{M} \sigma H=0
$$

Using now Eq. (5), we deduce

$$
\bar{R}(U, V) N=-(\nabla A)(U, V)+(\nabla A)(V, U), \quad U, V \in \mathfrak{X}(M) .
$$

On the other hand, the curvature tensor $R$ of $M$ is given as

$$
R(U, V) W=[\bar{R}(U, V) W]^{T}+g(A V, W) A U-g(A U, W) A V,
$$

where $[\bar{R}(U, V) W]^{T}$ is the tangential component of $\bar{R}(U, V) W$ to the hypersurface. If we choose a local orthonormal frame $\left\{e_{1}, \ldots, e_{2 n}\right\}$ on $M$ and use Eq. (17), we derive that the Ricci tensor Ric of $M$ has the next expression

$$
\operatorname{Ric}(U, V)=2 n H g(A U, V)-g(A U, A V)+\sum_{i=1}^{2 n} \bar{R}\left(e_{i}, U ; V, e_{i}\right)
$$

Note that

$$
\sum_{i=1}^{2 n} \bar{R}\left(e_{i}, U ; V, e_{i}\right)=\overline{\operatorname{Ric}}(U, V)-\bar{R}(N, U ; V, N)
$$

and therefore we deduce

$$
\operatorname{Ric}(U, V)=2 n H g(A U, V)-g(A U, A V)+\overline{\operatorname{Ric}}(U, V)-\bar{R}(N, U ; V, N) .
$$

Also, note that, on an orientable hypersurface $M$ of a Sasakian manifold $\bar{M}(\varphi, \xi, \eta, g)$ of dimension $(2 n+1)$, one can consider two globally defined orthogonal vector fields $\mathbf{u}, \mathbf{v}$ and they span plane sections of the tangent bundle of $M$. Recall now that the sectional curvature $K(\mathbf{u}, \mathbf{v})$ is defined as

$$
K(\mathbf{u}, \mathbf{v})=\frac{R(\mathbf{u}, \mathbf{v} ; \mathbf{v}, \mathbf{u})}{\|\mathbf{u}\|^{2}\|\mathbf{v}\|^{2}}
$$

Next, we recall that if $f$ is a smooth function on a Riemannian manifold $(M, g)$, then the Hessian operator $A_{f}$ is a symmetric operator defined by

$$
A_{f} U=\nabla_{U} \nabla f, \quad U \in \mathfrak{X}(M),
$$


and the Laplace operator $\Delta$ acting on smooth functions of $M$ is defined as

$$
\Delta f=\operatorname{div}(\nabla f) .
$$

Moreover, we have $\Delta f=\operatorname{tr} A_{f}$. We also denote by the same symbol, $\Delta$, the Laplace operator acting on smooth vector fields on $M$ defined by

$$
\Delta X=\sum_{i=1}^{n}\left(\nabla_{e_{i}} \nabla_{e_{i}} X-\nabla_{\nabla_{e_{i}} e_{i}} X\right), \quad X \in \mathfrak{X}(M)
$$

where $\left\{e_{1}, \ldots, e_{n}\right\}$ is a local orthonormal frame on $M, n=\operatorname{dim} M$. A vector field $X$ is said to be an eigenvector of $\Delta$ with eigenvalue $\lambda$ if $\Delta X=-\lambda X$. If $\lambda=0, X$ is said to be harmonic vector field.

\section{Totally umbilic hypersurfaces}

As we have noticed in the introduction, the most relevant results on hypersurfaces of a Sasakian manifold concern totally umbilical hypersurfaces. Therefore, it becomes an interesting question to seek conditions which are necessary and sufficient for a hypersurface of a Sasakian manifold to be totally umbilical. In the first result of this section we answer this question for a compact hypersurface of a Sasakian manifold and observe that sectional curvatures of the plane sections spanned by $\{\mathbf{u}, \mathbf{v}\}$ and the volume of the hypersurface play an important role. We also find a necessary condition for a compact hypersurface of a Sasakian manifold to be a CMC-hypersurface.

Theorem 3.1 A compact and connected orientable hypersurface $M$ of a $(2 n+1)$ dimensional Sasakian manifold $\bar{M}(\varphi, \xi, \eta, g)$ with sectional curvature $K(\mathbf{u}, \mathbf{v})>0$ and mean curvature $H$ constant along the integral curves of $\mathbf{v}$ is a totally umbilical hypersurface with constant mean curvature if and only if its volume $\boldsymbol{V}(M)$ satisfies

$$
\boldsymbol{V}(M) \leq \frac{1}{n-1} \int_{M}\left(\frac{1}{2} \operatorname{Ric}(\mathbf{v}, \mathbf{v})-\left(n(2 n-1) H^{2}+1\right) \sigma^{2}\right) .
$$

Proof Suppose $M$ is a compact hypersurface with $K(\mathbf{u}, \mathbf{v})>0$ and the mean curvature $H$ is constant along the integral curves of $\mathbf{v}$ and the volume of $M$ satisfies (19). Then choosing a local orthonormal frame $\left\{e_{1}, \ldots, e_{2 n}\right\}$ on the hypersurface, and using Eq. (16), we have for any vector field $U$ tangent to $M$

$$
\begin{aligned}
2 n U(H) & =\sum_{i=1}^{2 n} g\left((\nabla A)\left(U, e_{i}\right), e_{i}\right) \\
& =\sum_{i=1}^{2 n} g\left((\nabla A)\left(e_{i}, U\right)+\bar{R}\left(e_{i}, U\right) N, e_{i}\right) \\
& =\overline{\operatorname{Ric}}(U, N)+g\left(U, \sum_{i=1}^{2 n}(\nabla A)\left(e_{i}, e_{i}\right)\right) .
\end{aligned}
$$

Now, using Eq. (14), we have

$$
\operatorname{div}(H \mathbf{v})=\mathbf{v}(H)+2 n \sigma H^{2},
$$


which in view of Eq. (20) implies

$$
2 n \operatorname{div}(H \mathbf{v})=\overline{\operatorname{Ric}}(\mathbf{v}, N)+g\left(\mathbf{v}, \sum_{i=1}^{2 n}(\nabla A)\left(e_{i}, e_{i}\right)\right)+(2 n)^{2} \sigma H^{2} .
$$

Also, using the above equation in

$$
\operatorname{div}(\sigma H \mathbf{v})=H \mathbf{v}(\sigma)+\sigma \operatorname{div}(H \mathbf{v}),
$$

we conclude

$$
\begin{aligned}
2 n \operatorname{div}(\sigma H \mathbf{v})= & 2 n H g(-A \mathbf{v}, \mathbf{v})+\sigma \overline{\operatorname{Ric}}(\mathbf{v}, N) \\
& +\sigma g\left(\mathbf{v}, \sum_{i=1}^{2 n}(\nabla A)\left(e_{i}, e_{i}\right)\right)+(2 n)^{2} \sigma^{2} H^{2},
\end{aligned}
$$

where we have used $\nabla \sigma=-A \mathbf{v}-\mathbf{u}$. Thus, we have

$$
\begin{aligned}
\sigma g\left(\mathbf{v}, \sum_{i=1}^{2 n}(\nabla A)\left(e_{i}, e_{i}\right)\right)= & 2 n H g(A \mathbf{v}, \mathbf{v})-\sigma \overline{\operatorname{Ric}}(\mathbf{v}, N) \\
& -4 n^{2} \sigma^{2} H^{2}+2 n \operatorname{div}(\sigma H \mathbf{v}) .
\end{aligned}
$$

Next, we compute $\operatorname{div}(A \mathbf{v})$ with the aid of second equation in (12) and get

$$
\begin{aligned}
\operatorname{div}(A \mathbf{v}) & =\sum_{i=1}^{2 n} g\left(-F\left(e_{i}\right)+\sigma A e_{i}, A e_{i}\right)+g\left(\mathbf{v}, \sum_{i=1}^{2 n}(\nabla A)\left(e_{i}, e_{i}\right)\right) \\
& =\sigma\|A\|^{2}+g\left(\mathbf{v}, \sum_{i=1}^{2 n}(\nabla A)\left(e_{i}, e_{i}\right)\right),
\end{aligned}
$$

where we have used $\operatorname{tr} A \circ F=0$. Thus, we derive

$$
\operatorname{div}(\sigma A \mathbf{v})=g(\nabla \sigma, A \mathbf{v})+\sigma^{2}\|A\|^{2}+\sigma g\left(\mathbf{v}, \sum_{i=1}^{2 n}(\nabla A)\left(e_{i}, e_{i}\right)\right),
$$

and using $\nabla \sigma=-A \mathbf{v}-\mathbf{u}$ we conclude

$$
\operatorname{div}(\sigma A \mathbf{v})=-\|A \mathbf{v}\|^{2}-g(A \mathbf{v}, \mathbf{u})+\sigma^{2}\|A\|^{2}+\sigma g\left(\mathbf{v}, \sum_{i=1}^{2 n}(\nabla A)\left(e_{i}, e_{i}\right)\right) .
$$

Inserting Eq. (21) in the above equation, we get

$$
\begin{aligned}
& \operatorname{div}(\sigma A \mathbf{v})-2 n \operatorname{div}(\sigma H \mathbf{v})=-\|A \mathbf{v}\|^{2}-g(A \mathbf{v}, \mathbf{u})+\sigma^{2}\|A\|^{2}+2 n H g(A \mathbf{v}, \mathbf{v}) \\
& -\sigma \overline{\operatorname{Ric}}(\mathbf{v}, N)-4 n^{2} \sigma^{2} H^{2} .
\end{aligned}
$$

Now, using Eq. (18), we have

$$
\operatorname{Ric}(\mathbf{v}, \mathbf{v})=2 n H g(A \mathbf{v}, \mathbf{v})-\|A \mathbf{v}\|^{2}+\overline{\operatorname{Ric}}(\mathbf{v}, \mathbf{v})-\bar{R}(N, \mathbf{v} ; \mathbf{v}, N) .
$$


Note that $\mathbf{v}=\xi-\sigma N$, and using Eq. (4), we get

$$
\overline{\operatorname{Ric}}(\mathbf{v}, \mathbf{v})=2 n-4 n \sigma^{2}+\sigma^{2} \overline{\operatorname{Ric}}(N, N)
$$

and

$$
\bar{R}(N, \mathbf{v} ; \mathbf{v}, N)=1-\sigma^{2} .
$$

Thus, we conclude

$$
\begin{aligned}
\operatorname{Ric}(\mathbf{v}, \mathbf{v})= & 2 n H g(A \mathbf{v}, \mathbf{v})-\|A \mathbf{v}\|^{2}+\sigma^{2} \overline{\operatorname{Ric}}(N, N) \\
& +(2 n-1)-(4 n-1) \sigma^{2} .
\end{aligned}
$$

Also, using

$$
\overline{\operatorname{Ric}}(\mathbf{v}, N)=2 n \sigma-\sigma \overline{\operatorname{Ric}}(N, N)
$$

in Eq. (23) and subtracting Eq. (24) from it, we get

$$
\begin{aligned}
\operatorname{div}(\sigma A \mathbf{v})-2 n \operatorname{div}(\sigma H \mathbf{v})= & -g(A \mathbf{v}, \mathbf{u})+\sigma^{2}\|A\|^{2}-4 n^{2} \sigma^{2} H^{2} \\
& +\operatorname{Ric}(\mathbf{v}, \mathbf{v})-(2 n-1)+(2 n-1) \sigma^{2} .
\end{aligned}
$$

Integrating the above equation, we get

$$
\int_{M}\left(\sigma^{2}\|A\|^{2}-4 n^{2} \sigma^{2} H^{2}+\operatorname{Ric}(\mathbf{v}, \mathbf{v})-(2 n-1)+(2 n-1) \sigma^{2}-g(A \mathbf{v}, \mathbf{u})\right)=0 .
$$

Note that using (14) and $\nabla \sigma=-A \mathbf{v}-\mathbf{u}$, we have

$$
\begin{aligned}
\operatorname{div}(\sigma \mathbf{u}) & =g(-A \mathbf{v}-\mathbf{u}, \mathbf{u})+\sigma(2 n \sigma) \\
& =-g(A \mathbf{v}, \mathbf{u})-\left(1-\sigma^{2}\right)+2 n \sigma^{2},
\end{aligned}
$$

that is,

$$
-g(A \mathbf{v}, \mathbf{u})=\operatorname{div}(\sigma \mathbf{u})+1-(2 n+1) \sigma^{2}
$$

Inserting this value in the above integral, we conclude

$$
\int_{M}\left(\sigma^{2}\|A\|^{2}-4 n^{2} \sigma^{2} H^{2}+\operatorname{Ric}(\mathbf{v}, \mathbf{v})-2(n-1)-2 \sigma^{2}\right)=0
$$

that is,

$$
\begin{aligned}
\int_{M} \sigma^{2}\left(\|A\|^{2}-2 n H^{2}\right)= & 2(n-1) \boldsymbol{V}(M) \\
& +\int_{M}\left(\left(2 n(2 n-1) H^{2}+2\right) \sigma^{2}-\operatorname{Ric}(\mathbf{v}, \mathbf{v})\right) .
\end{aligned}
$$


The above equation implies

$$
\begin{aligned}
\int_{M} \sigma^{2}\left(\|A\|^{2}-2 n H^{2}\right)= & 2(n-1) \boldsymbol{V}(M) \\
& -2 \int_{M}\left(\frac{1}{2} \operatorname{Ric}(\mathbf{v}, \mathbf{v})-\left(n(2 n-1) H^{2}+1\right) \sigma^{2}\right) .
\end{aligned}
$$

If the inequality (19) holds, then in view of Schwartz's inequality

$$
\|A\|^{2} \geq 2 n H^{2}
$$

and the above equation gives

$$
\sigma^{2}\left(\|A\|^{2}-2 n H^{2}\right)=0
$$

Supposing $\sigma=0$, then from Eqs. (8) and (9) it follows that $\{\mathbf{u}, \mathbf{v}\}$ is an orthonormal set and $F(\mathbf{u})=0, F(\mathbf{v})=0$. Thus, using Eq. (12), we have

$$
\nabla_{\mathbf{u}} \mathbf{v}=0, \quad \nabla_{\mathbf{v}} \mathbf{v}=0
$$

and

$$
R(\mathbf{u}, \mathbf{v}) \mathbf{v}=-\nabla_{[\mathbf{u}, \mathbf{v}]} \mathbf{v}=-F([\mathbf{u}, \mathbf{v}])
$$

Therefore, the sectional curvature $K(\mathbf{u}, \mathbf{v})$ is

$$
K(\mathbf{u}, \mathbf{v})=-g(F([\mathbf{u}, \mathbf{v}]), \mathbf{u})=0
$$

which is contrary to the assumption that $K(\mathbf{u}, \mathbf{v})>0$. Hence, $\sigma \neq 0$ and consequently, on connected $M$, Eq. (26) implies the equality $\|A\|^{2}=2 n H^{2}$. We know that this equality in Schwartz's inequality holds if and only if

$$
A=H I \text {, }
$$

where $I$ denotes the identity operator. Now, we proceed to show that $H$ is a constant. Note that, due to (27), Eqs. (12) take the form

$$
\nabla_{U} \mathbf{u}=\sigma U+H F(U), \quad \nabla_{U} \mathbf{v}=-F(U)+\sigma H U, \quad \nabla \sigma=-H \mathbf{v}-\mathbf{u} .
$$

Using the above equations, we compute the Hessian operator $A_{\sigma}$ and find

$$
A_{\sigma} U=-U(H) \mathbf{v}-\left(1+H^{2}\right) \sigma U
$$

As the Hessian operator $A_{\sigma}$ is symmetric, we conclude that

$$
U(H) g(\mathbf{v}, V)=V(H) g(\mathbf{v}, U), \quad U, V \in \mathfrak{X}(M) .
$$


Taking $V=\mathbf{v}$ and using the fact that $H$ is constant along the integral curves of $\mathbf{v}$, we conclude that $\|\mathbf{v}\|^{2} U(H)=0$. Note that $\|\mathbf{v}\|^{2}=0$ implies $\sigma= \pm 1$ and it gives a contradiction to Eq. (15). Hence, $U(H)=0$ for all $U \in \mathfrak{X}(M)$. Therefore, $H$ is a constant and we conclude that $M$ is a totally umbilical hypersurface with constant mean curvature.

Conversely, suppose $M$ is a totally umbilical hypersurface with constant mean curvature. Then $M$ is isometric to $\mathbf{S}^{2 n}\left(1+H^{2}\right)$ (cf. [34]). Moreover, it follows that the sectional curvature $K(\mathbf{u}, \mathbf{v})>0$ and the Ricci curvature

$$
\operatorname{Ric}(\mathbf{v}, \mathbf{v})=(2 n-1)\left(1+H^{2}\right)\|\mathbf{v}\|^{2}=(2 n-1)\left(1+H^{2}\right)\left(1-\sigma^{2}\right) .
$$

Note that Eqs. (25) and (27) give

$$
\int_{M} \sigma^{2}=\frac{1}{2 n+1} \boldsymbol{V}(M), \quad \int_{M}\left(1-\sigma^{2}\right)=\frac{2 n}{2 n+1} \boldsymbol{V}(M) .
$$

Now, integrating Eq. (28) and using the last equation, we get

$$
\frac{1}{2} \int_{M} \operatorname{Ric}(\mathbf{v}, \mathbf{v})=\frac{n(2 n-1)}{2 n+1}\left(1+H^{2}\right) \boldsymbol{V}(M) .
$$

Also, using Eq. (29), we have

$$
\int_{M}\left(n(2 n-1) H^{2}+1\right) \sigma^{2}=\frac{1}{2 n+1}\left(n(2 n-1) H^{2}+1\right) \boldsymbol{V}(M)
$$

and subtracting the above equation from Eq. (30), we conclude

$$
\int_{M}\left(\frac{1}{2} \operatorname{Ric}(\mathbf{v}, \mathbf{v})-\left(n(2 n-1) H^{2}+1\right) \sigma^{2}\right)=(n-1) \mathbf{V}(M) .
$$

Hence, we get the equality

$$
\boldsymbol{V}(M)=\frac{1}{n-1} \int_{M}\left(\frac{1}{2} \operatorname{Ric}(\mathbf{v}, \mathbf{v})-\left(n(2 n-1) H^{2}+1\right) \sigma^{2}\right)
$$

required in the statement. Thus, all the requirements are met.

Recall that a hypersurface of a Riemannian manifold having constant mean curvature is called a CMC-hypersurface. Moreover, CMC-hypersurfaces in unit spheres are of particular interest not only in geometry but also in theoretical physics. Since the odd dimensional unit sphere $\mathbf{S}^{2 n+1}$ is a Sasakian manifold, it is an interesting question to find necessary conditions for a compact hypersurface of a Sasakian manifold to be a CMC-hypersurface. We answer this question in the following.

Theorem 3.2 Let $M$ be a compact and connected orientable hypersurface of a $(2 n+1)$ dimensional Sasakian manifold $\bar{M}(\varphi, \xi, \eta, g)$ with mean curvature H. If Av $=\mu \mathbf{v}$ for a constant $\mu$ and $H \geq \mu$ holds, then $M$ is a CMC-hypersurface.

Proof Suppose $A \mathbf{v}=\mu \mathbf{v}$ for a constant $\mu$. Then Eq. (12) implies

$$
\nabla \sigma=-\mu \mathbf{v}-\mathbf{u}
$$


and consequently $\mathbf{v}(\sigma)=-\mu\|\mathbf{v}\|^{2}$. Now, using Eq. (14), we infer

$$
\operatorname{div}(\sigma \mathbf{v})=-\mu\|\mathbf{v}\|^{2}+2 n H \sigma^{2}=-\mu\left(1-\sigma^{2}\right)+2 n H \sigma^{2} .
$$

Integrating the above equation, we have

$$
\mu \boldsymbol{V}(M)=\int_{M}(2 n H+\mu) \sigma^{2} .
$$

Now, integrating Eq. (25) and using $A \mathbf{v}=\mu \mathbf{v}$, we obtain

$$
\int_{M} \sigma^{2}=\frac{1}{2 n+1} \boldsymbol{V}(M)
$$

Combining Eqs. (31) and (32), we conclude

$$
2 n \int_{M}(H-\mu) \sigma^{2}=0
$$

Since $H \geq \mu$, the integrand in the above integral is non-negative and we get

$$
(H-\mu) \sigma^{2}=0 .
$$

If $\sigma^{2}=0$, then Eqs. (8) and (12) imply $\|\mathbf{u}\|^{2}=1$ and $\mu \mathbf{v}=-\mathbf{u}$. Taking the inner product in the last equation with $\mathbf{u}$, we get a contradiction, namely $\|\mathbf{u}\|^{2}=0$. Hence, $\sigma^{2} \neq 0$ and Eq. (33) on connected $M$ implies $H=\mu$, that is, $H$ is a constant.

Finally, for compact hypersurfaces of the unit sphere $\mathbf{S}^{2 n+1}$, we have the following characterization of small spheres in $\mathbf{S}^{2 n+1}$.

Theorem 3.3 A compact and connected hypersurface $M$ of the unit sphere $\mathbf{S}^{2 n+1}$ with mean curvature $H$ and $A \mathbf{v}=\mu \mathbf{v}$ for a constant $\mu \leq H$, is isometric to the sphere $\mathbf{S}^{2 n}\left(1+\mu^{2}\right)$ if and only if its volume satisfies

$$
\boldsymbol{V}(M) \leq \frac{2 n+1}{2 n(2 n-1)\left(1+H^{2}\right)} \int_{M} \operatorname{Ric}(\mathbf{v}, \mathbf{v}) .
$$

Proof Recall that compact hypersurfaces of the unit sphere $\mathbf{S}^{2 n+1}$ are orientable. Suppose $M$ is a compact hypersurface of $\mathbf{S}^{2 n+1}$ that satisfies the conditions in the statement of theorem. Then by Theorem 3.2, $M$ is a CMC-hypersurface, that is, $H$ is a constant and $H=\mu$. Now, using integral equation involving $\boldsymbol{V}(M)$ just before Eq. (26), we have

$$
\begin{aligned}
\int_{M} \sigma^{2}\left(\|A\|^{2}-2 n H^{2}\right)= & 2(n-1) \boldsymbol{V}(M) \\
& +2\left[n(2 n-1) H^{2}+1\right] \int_{M} \sigma^{2}-\int_{M} \operatorname{Ric}(\mathbf{v}, \mathbf{v}) .
\end{aligned}
$$

Using Eq. (32) in the above equation, we get

$$
\int_{M} \sigma^{2}\left(\|A\|^{2}-2 n H^{2}\right)=\frac{2 n(2 n-1)}{2 n+1}\left(1+H^{2}\right) \boldsymbol{V}(M)-\int_{M} \operatorname{Ric}(\mathbf{v}, \mathbf{v}) .
$$


Using the upper bound on $\boldsymbol{V}(M)$, we conclude

$$
\int_{M} \sigma^{2}\left(\|A\|^{2}-2 n H^{2}\right) \leq 0
$$

and as the integrand is non-negative, the above inequality gives

$$
\sigma^{2}\left(\|A\|^{2}-2 n H^{2}\right)=0 \text {. }
$$

However, because $\sigma^{2} \neq 0$, as noticed in the proof of Theorem 3.2, we get $\|A\|^{2}=2 n H^{2}$ and consequently $A=H I$. Hence, $M$ is totally umbilical hypersurface of constant mean curvature $H=\mu$ and thus, $M$ is isometric to the sphere $\mathbf{S}^{2 n}\left(1+\mu^{2}\right)$.

Conversely, if we suppose the hypersurface $M$ is isometric to $\mathbf{S}^{2 n}\left(1+\mu^{2}\right)$, then $M$ is a totally umbilic hypersurface with $A=\mu I$ and the mean curvature $H=\mu$. Then we have

$$
\operatorname{Ric}(\mathbf{v}, \mathbf{v})=(2 n-1)\left(1+H^{2}\right)\|\mathbf{v}\|^{2}=(2 n-1)\left(1+H^{2}\right)\left(1-\sigma^{2}\right) .
$$

Integrating the above equation and using Eq. (32), we get

$$
\int_{M} \operatorname{Ric}(\mathbf{v}, \mathbf{v})=\frac{2 n(2 n-1)}{2 n+1}\left(1+H^{2}\right) \mathbf{v}(M),
$$

that is,

$$
\boldsymbol{V}(M)=\frac{2 n+1}{2 n(2 n-1)\left(1+H^{2}\right)} \int_{M} \operatorname{Ric}(\mathbf{v}, \mathbf{v})
$$

and the proof is now complete.

\section{Vector fields $u$ and $v$ as eigenvectors of Laplace operator}

Let $M$ be a totally geodesic hypersurface of a $(2 n+1)$-dimensional Sasakian manifold $\bar{M}(\varphi, \xi, \eta, g)$. Then Eq. (12) takes the form

$$
\nabla_{U} \mathbf{u}=\sigma U, \quad \nabla_{U} \mathbf{v}=-F(U), \quad \nabla \sigma=-\mathbf{u}, \quad U \in \mathfrak{X}(M),
$$

and using these equations and Eq. (13), we get

$$
\Delta \mathbf{u}=-\mathbf{u}, \quad \Delta \mathbf{v}=-(2 n-1) \mathbf{v} .
$$

Thus, we see that both vector fields $\mathbf{u}, \mathbf{v}$ are eigenvectors of the Laplace operator on the totally geodesic hypersurface $M$. However, we see that if $M$ is totally umbilic hypersurface with constant mean curvature $H$, then we get

$$
\Delta \mathbf{u}=-\left(1+(2 n-1) H^{2}\right) \mathbf{u}+2(n-1) H \mathbf{v}, \quad \Delta \mathbf{v}=-\left(2 n-1+H^{2}\right) \mathbf{v}-2 n H \mathbf{u},
$$

that is, $\mathbf{u}, \mathbf{v}$ are not eigenvectors of the Laplace operator on non-totally geodesic totally umbilical hypersurface $M$. This raises a question: Do vectors $\mathbf{u}, \mathbf{v}$ as eigenvectors of Laplace operator on compact hypersurfaces of a Sasakian manifold can be used to characterize totally geodesic hypersurfaces? In this section, we answer this question. 
Theorem 4.1 Let $M$ be a compact and connected orientable hypersurface of $a(2 n+1)$ dimensional Sasakian manifold $\bar{M}(\varphi, \xi, \eta, g)$ with $\Delta \mathbf{u}=-\lambda \mathbf{u}$ for a positive constant $\lambda$. Then $M$ is totally geodesic if and only if its volume satisfies

$$
\boldsymbol{V}(M) \leq \frac{1}{\lambda} \int_{M}\left((2 n+\lambda) \sigma^{2}-\|A \mathbf{u}\|^{2}-\|A \mathbf{v}\|^{2}\right) .
$$

Proof Let $M$ be a compact and connected orientable hypersurface of a $(2 n+1)$ dimensional Sasakian manifold $\bar{M}(\varphi, \xi, \eta, g)$ and $\Delta \mathbf{u}=-\lambda \mathbf{u}$ for a positive constant $\lambda$. Then taking a local orthonormal frame $\left\{e_{1}, \ldots, e_{2 n}\right\}$ on $M$ and using Eq. (12), we compute

$$
\Delta \mathbf{u}=\sum_{i=1}^{2 n}\left[e_{i}(\sigma) e_{i}+(\nabla F)\left(e_{i}, A e_{i}\right)+F\left((\nabla A)\left(e_{i}, e_{i}\right)\right)\right] .
$$

Using Eq. (13) and $\Delta \mathbf{u}=-\lambda \mathbf{u}$ in the above equation, we have

$$
-\lambda \mathbf{u}=\nabla \sigma+2 n H \mathbf{v}-A \mathbf{v}+A^{2} \mathbf{u}-\|A\|^{2} \mathbf{u}+F\left(\sum_{i=1}^{2 n}(\nabla A)\left(e_{i}, e_{i}\right)\right) .
$$

Now, substituting $-A \mathbf{v}=\nabla \sigma+\mathbf{u}$ in the above equation and taking the inner product with $\mathbf{u}$, we conclude

$$
\left(-\lambda-1+\|A\|^{2}\right)\|\mathbf{u}\|^{2}=2 \mathbf{u}(\sigma)+\|A \mathbf{u}\|^{2}+\sigma g\left(\mathbf{v}, \sum_{i=1}^{2 n}(\nabla A)\left(e_{i}, e_{i}\right)\right),
$$

where we have used $F(\mathbf{u})=-\sigma \mathbf{v}$ (cf. Eq. (9)). Now, using Eq. (22), we have

$$
\sigma g\left(\mathbf{v}, \sum_{i=1}^{2 n}(\nabla A)\left(e_{i}, e_{i}\right)\right)=\|A \mathbf{v}\|^{2}+g(A \mathbf{v}, \mathbf{u})-\sigma^{2}\|A\|^{2}+\operatorname{div}(\sigma A \mathbf{v})
$$

and substituting this value in Eq. (36) yields

$$
\begin{aligned}
\left(-\lambda-1+\|A\|^{2}\right)\|\mathbf{u}\|^{2}= & 2 \mathbf{u}(\sigma)+\|A \mathbf{u}\|^{2}+\|A \mathbf{v}\|^{2} \\
& +g(A \mathbf{v}, \mathbf{u})-\sigma^{2}\|A\|^{2}+\operatorname{div}(\sigma A \mathbf{v}) .
\end{aligned}
$$

Using $\|\mathbf{u}\|^{2}=1-\sigma^{2}$ and $\mathbf{u}(\sigma)=\operatorname{div}(\sigma \mathbf{u})-2 n \sigma^{2}$ in the above equation and then integrating, we get

$$
\int_{M}\|A\|^{2}=\int_{M}\left(\|A \mathbf{u}\|^{2}+\|A \mathbf{v}\|^{2}+g(A \mathbf{v}, \mathbf{u})+(1+\lambda)\left(1-\sigma^{2}\right)-4 n \sigma^{2}\right) .
$$

Now, using Eq. (25) in the above equation, we get

$$
\int_{M}\|A\|^{2}=\lambda\left(\boldsymbol{V}(M)-\frac{1}{\lambda} \int_{M}\left((2 n+\lambda) \sigma^{2}-\|A \mathbf{u}\|^{2}-\|A \mathbf{v}\|^{2}\right)\right) .
$$

Using the volume estimate in the statement, we get $A=0$, that is, $M$ is totally geodesic. 
Conversely, suppose $M$ is a totally geodesic hypersurface of the Sasakian manifold $\bar{M}(\varphi, \xi, \eta, g)$. Then by Eq. (35), we see that $\Delta \mathbf{u}=-\lambda \mathbf{u}$, where $\lambda=1$. Moreover, integrating Eq. (25), in view of $A=0$, gives

$$
\boldsymbol{V}(M)=\frac{1}{\lambda} \int_{M}(2 n+\lambda) \sigma^{2},
$$

where $\lambda=1$, which is the equality case of the requirement in the statement.

Note that by Eq. (34) for a compact totally geodesic hypersurfaces of a $(2 n+1)$ dimensional Sasakian manifold $\bar{M}(\varphi, \xi, \eta, g)$, we have

$$
\nabla_{U} \nabla \sigma=-\sigma U, \quad U \in \mathfrak{X}(M) .
$$

If $\sigma$ is a constant, Eq. (14) implies $\sigma=0$. Then the equation $\|\mathbf{u}\|^{2}=1-\sigma^{2}=1$ implies $\mathbf{u}$ is a unit vector field. However, for constant $\sigma$, Eq. (34) implies $\mathbf{u}=0$ and we get a contradiction. Hence, Eq. (37) is Obata's differential equation (cf. [25, 31]) and, therefore, $M$ is isometric to the unit sphere $\mathbf{S}^{2 n}$. Recall that an odd dimensional unit sphere $\mathbf{S}^{2 n+1}$ as a real hypersurface of the complex space $\mathbf{C}^{n+1}$ has the standard Sasakian structure $(\varphi, \xi, \eta, g)$ [32]. As a particular case of the above theorem, we have the following consequence.

Corollary 4.2 Let $M$ be a compact and connected hypersurface of the unit sphere $\mathbf{S}^{2 n+1}$ with $\Delta \mathbf{u}=-\lambda \mathbf{u}$ for a positive constant $\lambda$. Then $M$ is isometric to the unit sphere $\mathbf{S}^{2 n}$ if and only if the volume of $M$ satisfies

$$
\boldsymbol{V}(M) \leq \frac{1}{\lambda} \int_{M}\left((2 n+\lambda) \sigma^{2}-\|A \mathbf{u}\|^{2}-\|A \mathbf{v}\|^{2}\right) .
$$

Theorem 4.3 Let $M$ be a compact and connected orientable hypersurface of a $(2 n+1)$ dimensional Sasakian manifold $\bar{M}(\varphi, \xi, \eta, g)$ such that the sectional curvature $K(\mathbf{u}, \mathbf{v})>0$ and $\Delta \mathbf{v}=-\lambda \mathbf{v}$ for a positive constant $\lambda$. Then $M$ is totally geodesic if and only if its volume satisfies

$$
\frac{(\lambda+2-2 n)}{\lambda+2} \boldsymbol{V}(M) \leq \int_{M} \sigma^{2} .
$$

Proof Using Eq. (1), we have

$$
\nabla_{U} \nabla_{U} \mathbf{v}-\nabla_{\nabla_{U} U} \mathbf{v}=-(\nabla F)(U, U)+U(\sigma) A U+\sigma(\nabla A)(U, U)
$$

and thus, choosing a local orthonormal frame $\left\{e_{1}, \ldots, e_{2 n}\right\}$ on $M$ and using Eq. (12), we compute

$$
\Delta \mathbf{v}=-((2 n-1) \mathbf{v}+A \mathbf{u}-2 n H \mathbf{u})+A \nabla \sigma+\sigma \sum_{i=1}^{n}(\nabla A)\left(e_{i}, e_{i}\right) .
$$


Using $\Delta \mathbf{v}=-\lambda \mathbf{v}$ in the above equation and taking the inner product with $\mathbf{v}$, we conclude

$$
\begin{aligned}
(2 n-1-\lambda)\|\mathbf{v}\|^{2}= & -g(A \mathbf{v}, \mathbf{u})+g(\nabla \sigma, A \mathbf{v}) \\
& +\sigma g\left(\mathbf{v}, \sum_{i=1}^{n}(\nabla A)\left(e_{i}, e_{i}\right)\right) .
\end{aligned}
$$

Now, using Eq. (22), we have

$$
\sigma g\left(\mathbf{v}, \sum_{i=1}^{2 n}(\nabla A)\left(e_{i}, e_{i}\right)\right)=\|A \mathbf{v}\|^{2}+g(A \mathbf{v}, \mathbf{u})-\sigma^{2}\|A\|^{2}+\operatorname{div}(\sigma A \mathbf{v})
$$

and using $\nabla \sigma=-A \mathbf{v}-\mathbf{u}$, that is,

$$
-g(\nabla \sigma, A \mathbf{v})=\|A \mathbf{v}\|^{2}+g(A \mathbf{v}, \mathbf{u})
$$

the above equation becomes

$$
\sigma g\left(\mathbf{v}, \sum_{i=1}^{2 n}(\nabla A)\left(e_{i}, e_{i}\right)\right)=-g(\nabla \sigma, A \mathbf{v})-\sigma^{2}\|A\|^{2}+\operatorname{div}(\sigma A \mathbf{v}) .
$$

Substituting the above relation in Eq. (38), we get

$$
(2 n-1-\lambda)\|\mathbf{v}\|^{2}=-g(A \mathbf{v}, \mathbf{u})-\sigma^{2}\|A\|^{2}+\operatorname{div}(\sigma A \mathbf{v}) .
$$

Now, integrating the above equation while using $\|\mathbf{v}\|^{2}=1-\sigma^{2}$ and Eq. (25), we conclude

$$
\int_{M} \sigma^{2}\|A\|^{2}=\int_{M}\left(\lambda+2-2 n-(\lambda+2) \sigma^{2}\right),
$$

that is,

$$
\int_{M} \sigma^{2}\|A\|^{2}=(\lambda+2)\left(\frac{\lambda+2-2 n}{\lambda+2} \boldsymbol{V}(M)-\int_{M} \sigma^{2}\right) .
$$

As the volume satisfies the condition given in the statement, and since $\sigma^{2}\|A\|^{2} \geq 0$, the above equation implies

$$
\sigma^{2}\|A\|^{2}=0
$$

If $\sigma^{2}=0$, then the set $\{\mathbf{u}, \mathbf{v}\}$ is an orthonormal set and we have seen in the proof of Theorem 3.1 that in this case the sectional curvature $K(\mathbf{u}, \mathbf{v})=0$ and this is contrary to our assumption that $K(\mathbf{u}, \mathbf{v})>0$. Hence, Eq. (39) on connected $M$ gives $A=0$.

Conversely, suppose $M$ is a compact connected orientable totally geodesic hypersurface of the Sasakian manifold $\bar{M}(\varphi, \xi, \eta, g)$. Then by Eq. (35), we have $\Delta \mathbf{v}=-\lambda \mathbf{v}$, where $\lambda=$ $2 n-1$. Moreover, using Eqs. (34) and (9), we have

$$
\nabla_{\mathbf{u}} \mathbf{u}=\sigma \mathbf{u}, \quad \nabla_{\mathbf{u}} \mathbf{v}=\sigma \mathbf{v}, \quad \nabla_{\mathbf{v}} \mathbf{v}=-\sigma \mathbf{u}, \quad \nabla_{\mathbf{v}} \mathbf{u}=\sigma \mathbf{v}, \quad \nabla \sigma=-\mathbf{u},
$$


and we get $[\mathbf{u}, \mathbf{v}]=0$, and also

$$
R(\mathbf{u}, \mathbf{v}) \mathbf{v}=\left(-\mathbf{u}(\sigma) \mathbf{u}-\sigma^{2} \mathbf{u}\right)-\left(\mathbf{v}(\sigma) \mathbf{v}-\sigma^{2} \mathbf{u}\right)=\|\mathbf{u}\|^{2} \mathbf{u} .
$$

Thus, $K(\mathbf{u}, \mathbf{v})=1>0$. Finally, for a totally geodesic hypersurface, (25) gives

$$
\int_{M} \sigma^{2}=\frac{1}{2 n+1} \boldsymbol{V}(M)
$$

that is,

$$
\frac{\lambda+2-2 n}{\lambda+2} \boldsymbol{V}(M)=\int_{M} \sigma^{2}
$$

Hence, all conditions are met.

Corollary 4.4 Let $M$ be a compact and connected hypersurface of the unit sphere $\mathbf{S}^{2 n+1}$ with sectional curvature $K(\mathbf{u}, \mathbf{v})>0$ and $\Delta \mathbf{v}=-\lambda \mathbf{v}$ for a positive constant $\lambda$. Then $M$ is isometric to the unit sphere $\mathbf{S}^{2 n}$ if and only if the volume of $M$ satisfies

$$
\frac{(\lambda+2-2 n)}{\lambda+2} \boldsymbol{V}(M) \leq \int_{M} \sigma^{2}
$$

\section{Bounds on Ricci curvature of hypersurfaces}

In this section, we use a bound on integrals of Ricci curvatures $\operatorname{Ric}(\mathbf{u}, \mathbf{u})$ to find conditions under which a compact and connected oriented hypersurface a $(2 n+1)$-dimensional Sasakian manifold $\bar{M}(\varphi, \xi, \eta, g)$ is isometric to a sphere. More interesting is the case of such hypersurfaces in the sphere $\mathbf{S}^{2 n+1}$, where the converse too holds. For a hypersurface $M$, we define a skew-symmetric operator $\Gamma=F \circ A+A \circ F$. Then, for a local orthonormal frame $\left\{e_{1}, \ldots, e_{2 n}\right\}$ on $M$, we have

$$
\|\Gamma\|^{2}=\sum_{i=1}^{2 n} g\left(\Gamma\left(e_{i}\right), \Gamma\left(e_{i}\right)\right), \quad \operatorname{tr}(F \circ A)^{2}=\sum_{i=1}^{2 n} g\left((F \circ A)^{2}\left(e_{i}\right), e_{i}\right) .
$$

Lemma 5.1 Let $M$ be a compact orientable hypersurface of a $(2 n+1)$-dimensional Sasakian manifold $\bar{M}(\varphi, \xi, \eta, g)$. Then the operator $\Gamma=F \circ A+A \circ F$ satisfies

$$
\frac{1}{2}\|\Gamma\|^{2}=\|A\|^{2}-\|A \mathbf{u}\|^{2}-\|A \mathbf{v}\|^{2}-\operatorname{tr}(F \circ A)^{2} .
$$

Proof Choosing a local orthonormal frame $\left\{e_{1}, \ldots, e_{2 n}\right\}$ on $M$, we notice that

$$
\|\Gamma\|^{2}=\|F \circ A\|^{2}+\|A \circ F\|^{2}+2 \sum_{i=1}^{2 n} g\left(A F\left(e_{i}\right), F\left(A e_{i}\right)\right)
$$

and therefore

$$
\|\Gamma\|^{2}=\|F \circ A\|^{2}+\|A \circ F\|^{2}-2 \operatorname{tr}(F \circ A)^{2} .
$$


Observe that, using Eq. (11), we have

$$
\|F \circ A\|^{2}=\|A\|^{2}-\|A \mathbf{u}\|^{2}-\|A \mathbf{v}\|^{2} .
$$

Note that on an open subset $S$ of $M$, where vector fields $\mathbf{u}$ and $\mathbf{v}$ are non-zero, if we choose a unit vector field $e$ orthogonal to both $\mathbf{u}$ and $\mathbf{v}$, then Eq. (10) implies that $F^{2}(e)=-e$ and that we have $g(F(e), \mathbf{u})=\sigma g(e, \mathbf{v})=0$, where we used Eq. (9). Similarly, $g(F(e), \mathbf{v})=0$. Thus, $F(e)$ is a unit vector field orthogonal to both $\mathbf{u}$ and $\mathbf{v}$. Note that the set $S$ is non-empty, because otherwise we get $\sigma= \pm 1$, which gives a contradiction to Eq. (15). Choosing $\overline{\mathbf{u}}=\frac{\mathbf{u}}{\|\mathbf{u}\|}$ and $\overline{\mathbf{v}}=\frac{\mathbf{v}}{\|\mathbf{v}\|}$ on $S$, we get a local orthonormal frame $\left\{e_{1}, \ldots, e_{n-1}, F\left(e_{1}\right), \ldots, F\left(e_{n-1}\right), \overline{\mathbf{u}}, \overline{\mathbf{v}}\right\}$ and we compute $\|A \circ F\|^{2}$, using this frame. We get

$$
\begin{aligned}
\|A \circ F\|^{2}= & \sum_{i=1}^{n-1} g\left(A F\left(e_{i}\right), A F\left(e_{i}\right)\right)+\sum_{i=1}^{n-1} g\left(A F^{2}\left(e_{i}\right), A F^{2}\left(e_{i}\right)\right) \\
& +g(A F(\overline{\mathbf{u}}), A F(\overline{\mathbf{u}}))+g(A F(\overline{\mathbf{v}}), A F(\overline{\mathbf{v}})) .
\end{aligned}
$$

Using Eqs. (8) and (9), we have $F(\overline{\mathbf{u}})=-\sigma \overline{\mathbf{v}}, F(\overline{\mathbf{v}})=\sigma \overline{\mathbf{u}},\|\mathbf{u}\|=\|\mathbf{v}\|=\sqrt{1-\sigma^{2}}$. Thus, we conclude

$$
\begin{aligned}
\|A \circ F\|^{2}= & \sum_{i=1}^{n-1} g\left(A e_{i}, A e_{i}\right)+\sum_{i=1}^{n-1} g\left(A F\left(e_{i}\right), A F\left(e_{i}\right)\right) \\
& +\sigma^{2}(g(A \overline{\mathbf{u}}, A \overline{\mathbf{u}})+g(A \overline{\mathbf{v}}, A \overline{\mathbf{v}})) . \\
= & \|A\|^{2}-g(A \overline{\mathbf{u}}, A \overline{\mathbf{u}})-g(A \overline{\mathbf{v}}, A \overline{\mathbf{v}})+\sigma^{2}(g(A \overline{\mathbf{u}}, A \overline{\mathbf{u}})+g(A \overline{\mathbf{v}}, A \overline{\mathbf{v}})) \\
= & \|A\|^{2}-\|A \mathbf{u}\|^{2}-\|A \mathbf{v}\|^{2} .
\end{aligned}
$$

Combining this equation and Eq. (41) with Eq. (40), we get the result.

Theorem 5.2 Let $M$ be a compact and connected orientable hypersurface of a $(2 n+1)$ dimensional Sasakian manifold $\bar{M}(\varphi, \xi, \eta, g)$ with mean curvature $H$ constant along integral curves of $\mathbf{v}$. If the Ricci curvature Ric $(\mathbf{u}, \mathbf{u})$ satisfies

$$
\int_{M} \operatorname{Ric}(\mathbf{u}, \mathbf{u}) \geq \int_{M}\left(2 n\left((2 n-1) \sigma^{2}-H^{2}\right)+\frac{1}{2}\|\Gamma\|^{2}+\|A \mathbf{u}\|^{2}+\|A \mathbf{v}\|^{2}\right),
$$

then $H$ is a constant and $M$ is isometric to the sphere $\mathbf{S}^{2 n}\left(1+H^{2}\right)$.

Proof Using Eq. (12), we compute

$$
\begin{aligned}
R(U, V) \mathbf{u}= & (U(\sigma) V-V(\sigma) U)+(\nabla F)(U, A V)-(\nabla F)(U, A V) \\
& +F(\nabla A)(U, V)-(\nabla A)(V, U) .
\end{aligned}
$$

Note that Eq. (13) implies

$$
\begin{aligned}
(\nabla F)(U, A V)-(\nabla F)(U, A V)= & g(A \mathbf{v}, U) V-g(A \mathbf{v}, V) U \\
& +g(A \mathbf{u}, V) A U-g(A \mathbf{u}, U) A V,
\end{aligned}
$$


and inserting this equation in (42), keeping in view $\nabla \sigma+A \mathbf{v}=-\mathbf{u}$, we conclude

$$
\begin{aligned}
R(U, V) \mathbf{u}= & g(\mathbf{u}, V) U-g(\mathbf{u}, U) V+g(A \mathbf{u}, V) A U-g(A \mathbf{u}, U) A V \\
& +F(\nabla A)(U, V)-(\nabla A)(V, U) .
\end{aligned}
$$

Taking the trace in the above equation, we arrive at

$$
\begin{aligned}
\operatorname{Ric}(V, \mathbf{u})= & (2 n-1) \alpha(V)+2 n H g(A \mathbf{u}, V) \\
& -g(A \mathbf{u}, A V)+\sum_{i=1}^{2 n} g\left((\nabla A)\left(V, e_{i}\right), F\left(e_{i}\right)\right) \\
& -g\left(V, \sum_{i=1}^{2 n}(\nabla A)\left(e_{i}, F\left(e_{i}\right)\right)\right),
\end{aligned}
$$

where we used skew-symmetry of the operator $F$ and symmetry of the shape operator $A$. Observe that $\operatorname{tr}(A \circ F)=0$ and using Eq. (13), we have

$$
\begin{aligned}
\sum_{i=1}^{2 n} g\left((\nabla A)\left(V, e_{i}\right), F\left(e_{i}\right)\right) & =\sum_{i=1}^{2 n} V g\left(A e_{i}, F\left(e_{i}\right)\right)-\sum_{i=1}^{2 n} g\left(A e_{i},(\nabla F)\left(V, e_{i}\right)\right) \\
& =-\sum_{i=1}^{2 n} g\left(A e_{i}, g\left(V, e_{i}\right) \mathbf{v}-\beta\left(e_{i}\right) V+\alpha\left(e_{i}\right) A V-g\left(A V, e_{i}\right) \mathbf{u}\right) \\
& =0 .
\end{aligned}
$$

Thus, Eq. (43) implies

$$
\begin{aligned}
\operatorname{Ric}(\mathbf{u}, \mathbf{u})= & (2 n-1)\|\mathbf{u}\|^{2}+2 n H g(A \mathbf{u}, \mathbf{u})-\|A \mathbf{u}\|^{2} \\
& -g\left(\mathbf{u}, \sum_{i=1}^{2 n}(\nabla A)\left(e_{i}, F\left(e_{i}\right)\right)\right) .
\end{aligned}
$$

Now, using Eqs. (12) and (13), we compute

$$
\begin{aligned}
\operatorname{div}(F(A \mathbf{u}))= & \sum_{i=1}^{2 n} g\left(\nabla_{e_{i}} F(A \mathbf{u}), e_{i}\right)=-\sum_{i=1}^{2 n} e_{i} g\left(\mathbf{u}, A F\left(e_{i}\right)\right) \\
= & -\sum_{i=1}^{2 n}\left[g\left(\sigma e_{i}+F\left(A e_{i}\right), A F\left(e_{i}\right)\right)+g\left(\mathbf{u},(\nabla A)\left(e_{i}, F\left(e_{i}\right)+A(\nabla F)\left(e_{i}, e_{i}\right)\right)\right]\right. \\
= & \sum_{i=1}^{2 n} g\left((F \circ A)^{2}\left(e_{i}\right), e_{i}\right)-g\left(\mathbf{u}, \sum_{i=1}^{2 n}(\nabla A)\left(e_{i}, F\left(e_{i}\right)\right)\right) \\
& -\sum_{i=1}^{2 n} g(A \mathbf{u},(2 n-1) \mathbf{v}+A \mathbf{u}-2 n H \mathbf{u}) .
\end{aligned}
$$


Thus, we have

$$
\begin{aligned}
-g\left(\mathbf{u}, \sum_{i=1}^{2 n}(\nabla A)\left(e_{i}, F\left(e_{i}\right)\right)\right)= & -\operatorname{tr}(F \circ A)^{2}+(2 n-1) g(A \mathbf{u}, \mathbf{v})+\|A \mathbf{u}\|^{2} \\
& -2 n H g(A \mathbf{u}, \mathbf{u})+\operatorname{div}(F(A \mathbf{u})) .
\end{aligned}
$$

Inserting the above expression in Eq. (44), we get

$$
\operatorname{Ric}(\mathbf{u}, \mathbf{u})=(2 n-1)\|\mathbf{u}\|^{2}-\operatorname{tr}(F \circ A)^{2}+(2 n-1) g(A \mathbf{u}, \mathbf{v})+\operatorname{div}(F(A \mathbf{u})) .
$$

Integrating, we get

$$
\int_{M} \operatorname{Ric}(\mathbf{u}, \mathbf{u})=\int_{M}\left((2 n-1)\|\mathbf{u}\|^{2}-\operatorname{tr}(F \circ A)^{2}+(2 n-1) g(A \mathbf{u}, \mathbf{v})\right) .
$$

Using $\|\mathbf{u}\|^{2}=1-\sigma^{2}$ and Eq. (25), we get

$$
\int_{M}\left(\operatorname{Ric}(\mathbf{u}, \mathbf{u})+\operatorname{tr}(F \circ A)^{2}-2 n(2 n-1) \sigma^{2}\right)=0 .
$$

Using Lemma 5.1, the above equation can be rearranged as

$$
\begin{aligned}
\int_{M}\left(\|A\|^{2}-2 n H^{2}\right)= & \int_{M}\left(2 n\left((2 n-1) \sigma^{2}-H^{2}\right)+\frac{1}{2}\|\Gamma\|^{2}+\|A \mathbf{u}\|^{2}+\|A \mathbf{v}\|^{2}\right) \\
& -\int_{M} \operatorname{Ric}(\mathbf{u}, \mathbf{u}) .
\end{aligned}
$$

But the above equation in view of given condition implies

$$
\int_{M}\left(\|A\|^{2}-2 n H^{2}\right) \leq 0
$$

However, owing to Schwartz's inequality the integrand in the above inequality is nonnegative. Hence, we have $\|A\|^{2}=2 n H^{2}$ and this being the equality in Schwartz's inequality, we must have

$$
A=H I \text {. }
$$

Now, we proceed to show that $H$ is a constant. Observe that Eq. (12) in view of Eq. (45) takes the following form

$$
\nabla_{U} \mathbf{u}=\sigma U+H F(U), \quad \nabla_{U} \mathbf{v}=-F(U)+\sigma H U, \quad \nabla \sigma=-H \mathbf{v}-\mathbf{u} .
$$

Using the above equation, we compute the Hessian operator $A_{\sigma}$ and find

$$
A_{\sigma}(U)=-U(H) \mathbf{v}-\left(1+H^{2}\right) \sigma U, \quad U \in \mathfrak{X}(M) .
$$

As $A_{\sigma}$ is symmetric, we conclude from the above equation

$$
U(H) \beta(V)=V(H) \beta(U), \quad U, V \in \mathfrak{X}(M),
$$


that is, $U(H) \mathbf{v}=\beta(U) \nabla H$. Taking $U=\mathbf{v}$ and using the fact that $H$ is constant along the integral curves of $\mathbf{v}$, we get

$$
\|\mathbf{v}\|^{2} \nabla H=0
$$

If $\|\mathbf{v}\|^{2}=0$, we get $\sigma= \pm 1$ and it follows immediately that (15) gives a contradiction. Thus, the above equation gives $\nabla H=0$, that is, $H$ is a constant. Hence, $M$ is a totally umbilical hypersurface of constant mean curvature $H$ and, consequently, $M$ is isometric to the sphere $\mathbf{S}^{2 n}\left(1+H^{2}\right)$ (cf. [34]).

In the following result, we prove that, for compact and connected hypersurfaces of the sphere $\mathbf{S}^{2 n+1}$, the converse of the above theorem also holds.

Theorem 5.3 Let $M$ be a compact and connected hypersurface of the sphere $\mathbf{S}^{2 n+1}$ with mean curvature $H$ constant along integral curves of $\mathbf{v}$. Then the mean curvature $H$ is a constant and $M$ is isometric to the sphere $\mathbf{S}^{2 n}\left(1+H^{2}\right)$ if and only if the Ricci curvature $\operatorname{Ric}(\mathbf{u}, \mathbf{u})$ satisfies

$$
\int_{M} \operatorname{Ric}(\mathbf{u}, \mathbf{u}) \geq \int_{M}\left(2 n\left((2 n-1) \sigma^{2}-H^{2}\right)+\frac{1}{2}\|\Gamma\|^{2}+\|A \mathbf{u}\|^{2}+\|A \mathbf{v}\|^{2}\right) .
$$

Proof If the Ricci curvature $\operatorname{Ric}(\mathbf{u}, \mathbf{u})$ satisfies the given condition, then by the previous theorem $H$ is a constant and $M$ is isometric to the sphere $\mathbf{S}^{2 n}\left(1+H^{2}\right)$.

Conversely, suppose that hypersurface $M$ is isometric to the sphere $\mathbf{S}^{2 n}\left(1+H^{2}\right)$. Then $M$ is a totally umbilical hypersurface of $\mathbf{S}^{2 n+1}$ with constant mean curvature $H$, that is, $A=H I$ holds. Then using Eq. (12) in the form $\nabla \sigma=-H \mathbf{v}-\mathbf{u}$ and $\operatorname{div}(\mathbf{u})=2 n \sigma$ we derive

$$
\operatorname{div}(\sigma \mathbf{u})=-\|\mathbf{u}\|^{2}+2 n \sigma^{2}=-1+(2 n+1) \sigma^{2} .
$$

Integrating the above equation, we get

$$
\int_{M} \sigma^{2}=\frac{1}{2 n+1} \boldsymbol{V}(M)
$$

The Ricci curvature is

$$
\operatorname{Ric}(\mathbf{u}, \mathbf{u})=(2 n-1)\left(1+H^{2}\right)\|\mathbf{u}\|^{2}=(2 n-1)\left(1+H^{2}\right)\left(1-\sigma^{2}\right),
$$

which in view of Eq. (46) gives

$$
\int_{M} \operatorname{Ric}(\mathbf{u}, \mathbf{u})=\frac{2 n(2 n-1)\left(1+H^{2}\right)}{2 n+1} \boldsymbol{V}(M) .
$$

Also, $\Gamma=F \circ A+A \circ F$, gives $\Gamma(U)=2 H F(U)$ and consequently, using Eq. (11), we have

$$
\|\Gamma\|^{2}=4 H^{2}\left(2 n-\|\mathbf{u}\|^{2}-\|\mathbf{v}\|^{2}\right)=8 H^{2}\left(n-1+\sigma^{2}\right) .
$$

Moreover, we have

$$
\|A \mathbf{u}\|^{2}+\|A \mathbf{v}\|^{2}=2 H^{2}\left(1-\sigma^{2}\right) .
$$


Thus, using the above equation and Eq. (48), we have

$$
\int_{M}\left(2 n\left((2 n-1) \sigma^{2}-H^{2}\right)+\frac{1}{2}\|\Gamma\|^{2}+\|A \mathbf{u}\|^{2}+\|A \mathbf{v}\|^{2}\right)=\frac{2 n(2 n-1)\left(1+H^{2}\right)}{2 n+1} \boldsymbol{V}(M) .
$$

Using the above equation with Eq. (47), we conclude

$$
\int_{M} \operatorname{Ric}(\mathbf{u}, \mathbf{u})=\int_{M}\left(2 n\left((2 n-1) \sigma^{2}-H^{2}\right)+\frac{1}{2}\|\Gamma\|^{2}+\|A \mathbf{u}\|^{2}+\|A \mathbf{v}\|^{2}\right) .
$$

Hence, all the requirements are met.

In the next result, we consider a compact hypersurface $M$ with $c=\inf _{M} \overline{\operatorname{Ric}}(N, N)$ on $M$, $c>0$ and prove the following.

Theorem 5.4 Let $M$ be a compact and connected orientable hypersurface of a $(2 n+1)$ dimensional Sasakian manifold $\bar{M}(\varphi, \xi, \eta, g)$ with mean curvature $H$ constant along integral curves of $\mathbf{v}$. If the sectional curvature $K(\mathbf{u}, \mathbf{v})>0$ and $c=\inf _{M} \overline{\operatorname{Ric}}(N, N)>0$ satisfies

$$
\int_{M}\|\nabla \sigma\|^{2} \leq \int_{M}\left(c+2 n H^{2}\right) \sigma^{2}
$$

then $H$ is a constant and $M$ is isometric to the sphere $\mathbf{S}^{2 n}\left(1+H^{2}\right)$.

Proof As the mean curvature $H$ is constant along the integral curves of $\mathbf{v}$, using Eq. (16) and an orthonormal frame $\left\{e_{1}, \ldots, e_{2 n}\right\}$, we have

$$
0=2 n \mathbf{v}(H)=\sum_{i=1}^{2 n} g\left((\nabla A)\left(\mathbf{v}, e_{i}\right), e_{i}\right)=\sum_{i=1}^{2 n} g\left((\nabla A)\left(e_{i}, \mathbf{v}\right)+\bar{R}\left(e_{i}, \mathbf{v}\right) N, e_{i}\right),
$$

that is,

$$
\sum_{i=1}^{2 n} g\left(\mathbf{v},(\nabla A)\left(e_{i}, e_{i}\right)\right)=-\overline{R i c}(\mathbf{v}, N)
$$

Now, using Eq. (12), we compute

$$
\operatorname{div} A \mathbf{v}=\sigma\|A\|^{2}+\sum_{i=1}^{2 n} g\left(\mathbf{v},(\nabla A)\left(e_{i}, e_{i}\right)\right)
$$

where we have used $\operatorname{tr} F \circ A=0$. Using Eq. (49) in the above equation, we get

$$
\operatorname{div} A \mathbf{v}=\sigma\|A\|^{2}-\overline{\operatorname{Ric}}(\mathbf{v}, N) .
$$

Using the above equation and equations $\nabla \sigma=-A \mathbf{v}-\mathbf{u}$ and (14), we conclude

$$
\Delta \sigma=-\sigma\|A\|^{2}+\overline{\operatorname{Ric}}(\mathbf{v}, N)-2 n \sigma .
$$


Note that

$$
\overline{\operatorname{Ric}}(\mathbf{v}, N)=\overline{\operatorname{Ric}}(\xi-\sigma N, N)=2 n \sigma-\sigma \overline{\operatorname{Ric}}(N, N),
$$

and consequently, the above equation gives

$$
\Delta \sigma=-\sigma\|A\|^{2}-\sigma \overline{\operatorname{Ric}}(N, N)
$$

that is,

$$
\sigma \Delta \sigma=-\sigma^{2}\|A\|^{2}-\sigma^{2} \overline{\operatorname{Ric}}(N, N) .
$$

Integrating the above equation by parts, we conclude

$$
\int_{M}\|\nabla \sigma\|^{2}=\int_{M}\left(\sigma^{2}\|A\|^{2}+\sigma^{2} \overline{\operatorname{Ric}}(N, N)\right),
$$

or

$$
\int_{M} \sigma^{2}\left(\|A\|^{2}-2 n H^{2}\right) \leq \int_{M}\left(\|\nabla \sigma\|^{2}-\left(c+2 n H^{2}\right) \sigma^{2}\right) .
$$

If the given inequality in the statement holds, then owing to Schwartz's inequality $\|A\|^{2} \geq$ $2 n H^{2}$, and therefore the inequality (50) implies

$$
\sigma^{2}\left(\|A\|^{2}-2 n H^{2}\right)=0
$$

If $\sigma=0$, then as $K(\mathbf{u}, \mathbf{v})>0$, following the proof of Theorem 3.1, we get a contradiction. Hence, on connected $M$, we have $\|A\|^{2}=2 n H^{2}$, which, being exactly the equality in the Schwartz inequality, holds if and only if

$$
A=H I .
$$

Now, as $\mathbf{v}(H)=0$, again following the proof of Theorem 3.1, we get $H$ is a constant. Thus, $M$ is a totally umbilical hypersurface of constant mean curvature $H$ in the Sasakian manifold and consequently, $M$ is isometric to the sphere $\mathbf{S}^{2 n}\left(1+H^{2}\right)$.

Observe that if $M$ is a compact hypersurface of the odd dimensional unit sphere $\mathbf{S}^{2 n+1}$, then in this case, we have $c=2 n$ and we can state the following result.

Corollary 5.5 Let $M$ be a compact and connected hypersurface of the unit sphere $\mathbf{S}^{2 n+1}$ with mean curvature $H$ constant along integral curves of $\mathbf{v}$ and the sectional curvature $K(\mathbf{u}, \mathbf{v})>0$. Then the following inequality holds:

$$
\int_{M}\|\nabla \sigma\|^{2} \leq 2 n \int_{M}\left(1+H^{2}\right) \sigma^{2}
$$

if and only if $H$ is a constant and $M$ is isometric to the sphere $\mathbf{S}^{2 n}\left(1+H^{2}\right)$. 
Proof If the given condition holds, then by the previous theorem it follows that $M$ is isometric to $\mathbf{S}^{2 n}\left(1+H^{2}\right)$. Conversely, suppose $M$ is a compact and connected hypersurface of the unit sphere $\mathbf{S}^{2 n+1}$ that is isometric to $\mathbf{S}^{2 n}\left(1+H^{2}\right)$. Then $M$ is a totally umbilical hypersurface with $A=H I$ and using Eqs. (12) and (14), we have $\nabla \sigma=-H \mathbf{v}-\mathbf{u}$, where $H$ is a constant. Thus,

$$
\Delta \sigma=-2 n H^{2} \sigma-2 n \sigma=-2 n\left(1+H^{2}\right) \sigma
$$

and we get

$$
\int_{M}\|\nabla \sigma\|^{2}=2 n \int_{M}\left(1+H^{2}\right) \sigma^{2}
$$

\section{Acknowledgements}

This article is dedicated to the memory of Professor Aurel Bejancu (19.08.1946-03.04.2020).

\section{Funding}

The authors extend their appreciations to the Deanship of Scientific Research King Saud University for funding this work through research group no (RG-1440-142).

\section{Availability of data and materials}

Not applicable.

\section{Competing interests}

The authors declare that they have no competing interests.

\section{Authors' contributions}

All authors contributed equally to the writing of this paper. All authors read and approved the final manuscript.

\section{Author details}

'Department of Mathematics, College of Science, King Saud University, P.O. Box-2455, 11451, Riyadh, Saudi Arabia.

${ }^{2}$ Institute of Physical and Mathematical Sciences and IT, Immanuel Kant Baltic Federal University, A. Nevsky str. 14, 236016 Kaliningrad, Russia. ${ }^{3}$ Department of Cybernetics, Economic Informatics, Finance and Accountancy, Petroleum-Gas University of Ploieşti, Bd. Bucureşti 39, 100680 Ploieşti, Romania.

\section{Publisher's Note}

Springer Nature remains neutral with regard to jurisdictional claims in published maps and institutional affiliations.

Received: 29 September 2020 Accepted: 5 March 2021 Published online: 19 March 2021

\section{References}

1. Adachi, T., Kameda, M., Maeda, S.: Geometric meaning of Sasakian space form the view point of submanifold therory. Kodai Math. J. 33, 383-397 (2010)

2. Al-Solamy, F.R., Khan, M.A.: Semi-invariant warped product submanifolds of almost contact manifolds. J. Inequal. Appl. 2012, 127 (2012)

3. Ali, A., Alkhaldi, A.H., Pişcoran, L.-I., Ali, R.: Eigenvalue inequalities for the $p$-Laplacian operator on C-totally real submanifolds in Sasakian space forms. Appl. Anal. (2020). https://doi.org/10.1080/00036811.2020.1758307

4. Ali, A., Pişcoran, L.-I.: Geometric classification of warped products isometrically immersed into Sasakian space forms. Math. Nachr. 292(2), 234-251 (2019)

5. Alodan, H., Chen, B.-Y., Deshmukh, S., Villcu, G.-E.: A generalized Wintgen inequality for quaternionic CR-submanifolds. Rev. R. Acad. Cienc. Exactas Fís. Nat., Ser. A Mat. 114(3), 129 (2020)

6. Alodan, H., Deshmukh, S., Turki, B.N., Vilcu, G.-E.: Hypersurfaces of a Sasakian manifold. Mathematics 8, 877 (2020)

7. Barbosa, E.: On CMC free-boundary stable hypersurfaces in a Euclidean ball. Math. Ann. 372(1-2), 179-187 (2018)

8. Bejancu, A., Deshmukh, S.: Real hypersurfaces of $\mathrm{CP}^{n}$ with non-negative Ricci curvature. Proc. Am. Math. Soc. 124(1), 269-274 (1996)

9. Bellettini, C., Chodosh, O., Wickramasekera, N.: Curvature estimates and sheeting theorems for weakly stable CMC hypersurfaces. Adv. Math. 352, 133-157 (2019)

10. Besse, A.L.: Einstein Manifolds. Springer, Berlin (1987)

11. Bettiol, R., Piccione, P., Santoro, B.: Deformations of free boundary CMC hypersurfaces. J. Geom. Anal. 27(4), 3254-3284 (2017)

12. Biswas, I., Schumacher, G.: Vector bundles on Sasakian manifolds. Adv. Theor. Math. Phys. 14(2), 541-561 (2010)

13. Blair, D.E.: Riemannian Geometry of Contact and Symplectic Manifolds, 2nd edn. Birkhäuser, Boston (2010)

14. Boyer, C., Galicki, K.: On Sasakian-Einstein geometry. Int. J. Math. 11(7), 873-909 (2001)

15. Boyer, C., Galicki, K.: Sasakian Geometry. Oxford Mathematical Monographs. Oxford University Press, Oxford (2008) 
16. Boyer, C., Tonnesen-Friedman, C.: The Sasaki join, Hamiltonian 2-forms, and constant scalar curvature. J. Geom. Anal. 26(2), 1023-1060 (2016)

17. Chen, B.-Y.: Pseudo-Riemannian Geometry, $\delta$-Invariants and Applications. World Scientific, Hackensack (2011)

18. Chen, B.-Y.: Differential Geometry of Warped Product Manifolds and Submanifolds. World Scientific, Hackensack (2017)

19. Collins, T.C., Székelyhidi, G.: Sasaki-Einstein metrics and K-stability. Geom. Topol. 23, 1339-1413 (2019)

20. de Almeida, S.C., Brito, F.G.B., Scherfner, M., Weiss, S.: On CMC hypersurfaces in $S^{n+1}$ with constant Gauss-Kronecker curvature. Adv. Geom. 18(2), 187-192 (2018)

21. Deshmukh, S.: Real hypersurfaces in a Euclidean complex space form. Q. J. Math. 58, 303-307 (2007)

22. Deshmukh, S.: Real hypersurfaces of a complex space form. Monatshefte Math. 166, 93-106 (2012)

23. El Kacimi-Alaoui, A.: Opérateurs transversalement elliptiques sur un feuilletage riemannien et applications. Compos. Math. 73(1), 57-106 (1990)

24. Feng, K., Zheng, T.: Transverse fully nonlinear equations on Sasakian manifolds and applications. Adv. Math. 357, 106830 (2019) 59 pp.

25. Fetcu, D., Oniciuc, C.: Biharmonic hypersurfaces in Sasakian space forms. Differ. Geom. Appl. 27, 713-722 (2009)

26. Futaki, A., Ono, H., Wang, G.: Transverse Kähler geometry of Sasaki manifolds and toric Sasaki-Einstein manifolds. J. Differ. Geom. 83(3), 585-636 (2009)

27. García-Martínez, C., Herrera, J.: Rigidity and bifurcation results for CMC hypersurfaces in warped product spaces. J. Geom. Anal. 26(2), 1186-1201 (2016)

28. Gervasio, C., de Lima, E., de Lima, H.: Characterizations of complete CMC spacelike hypersurfaces satisfying an Okumura type inequality. Differ. Geom. Appl. 56, 295-307 (2018)

29. Ghosh, A., Sharma, R.: Sasakian manifolds with purely transversal Bach tensor. J. Math. Phys. 58(10)، 103502 (2017)

30. He, W., Sun, S.: Frankel conjecture and Sasaki geometry. Adv. Math. 291, 912-960 (2016)

31. Kimura, M., Maeda, S.: On real hypersurface of a complex projective space. Math. Z. 202, 299-312 (1989)

32. Lee, J.W., Lee, C.W., Vîlcu, G.-E.: Classification of Casorati ideal Legendrian submanifolds in Sasakian space forms. J. Geom. Phys. 155, $103768(2020)$

33. Maeda, S., Tanabe, H., Udagawa, S.: Generating curves of minimal ruled real hypersurfaces in a nonflat complex space form. Can. Math. Bull. 62, 383-392 (2019)

34. Niebergall, R., Ryan, P.J.: Real hypersurfaces in complex space forms, Tight and Taut submanifolds (T.E. Cecil and S.S. Chern, eds.). Cambridge University Press, 233-305 (1998) (1998)

35. Obata, M.: Conformal transformations of Riemannian manifolds. J. Differ. Geom. 4, 311-333 (1970)

36. Obata, M.: The conjectures about conformal transformations. J. Differ. Geom. 6, 247-258 (1971)

37. Perdomo, O., Tkachev, V.: Algebraic CMC hypersurfaces of order 3 in Euclidean spaces. J. Geom. 110(1), Paper No. 6, 7 pp. (2019)

38. Sasahara, T.: Ricci curvature of real hypersurfaces in non-flat complex space forms. Mediterr. J. Math. 15, $12(2018)$

39. Siddiqui, A.N., Shahid, M.H., Lee, J.W.: Geometric inequalities for warped product bi-slant submanifolds with a warping function. J. Inequal. Appl. 2018, 265 (2018)

40. Slesar, V., Visinescu, M., Vîlcu, G.-E.: Toric data, Killing forms and complete integrability of geodesics in Sasaki-Einstein spaces $Y^{p, q}$. Ann. Phys. 361, 548-562 (2015)

41. Smoczyk, K., Wang, G., Zhang, Y.: Sasaki-Ricci flow. Int. J. Math. 21(7), 951-969 (2010)

42. Uddin, S., Khan, K.A.: An inequality for contact CR-warped product submanifolds of nearly cosymplectic manifolds. J. Inequal. Appl. 2012, 304 (2012)

43. Uhlenbeck, K., Yau, S.-T.: On the existence of Hermitian-Yang-Mills connections in stable vector bundles. Commun Pure Appl. Math. 39(S1), S257-S293 (1986)

44. Wang, Y.: Cyclic h-parallel shape and Ricci operators on real hypersurfaces in two-dimensional nonflat complex space forms. Pac. J. Math. 302, 335-352 (2019)

45. Watanabe, Y.: Totally umbilical surfaces in normal contact Riemannian manifold. Kodai Math. Semin. Rep. 19, 474-487 (1967)

46. Yamaguchi, S.: On hypersurfaces in Sasakian manifolds. Kodai Math. Semin. Rep. 21, 64-72 (1969)

\section{Submit your manuscript to a SpringerOpen ${ }^{\circ}$ journal and benefit from:}

- Convenient online submission

- Rigorous peer review

- Open access: articles freely available online

- High visibility within the field

- Retaining the copyright to your article

Submit your next manuscript at $>$ springeropen.com 